\title{
Comparison of Effectiveness of Mulligan 'MWM' Technique versus Kaltenborn Mobilization Technique on Pain and End Range of Motion in Patients with Adhesive Capsulitis of Shoulder Joint: A Randomized Controlled Trial
}

\author{
Sumit Ragav and Anshika Singh
}

\begin{abstract}
Aim: The aim of this study was to find the comparative effect of mulligan mobilization with movement (MWM) technique versus Kaltenborn mobilization technique on pain, disability and end range of motion of shoulder joint in patients with adhesive capsulitis. Method: The study was a randomized controlled trial with a sample of 30 subjects, 19 were female, 11 were male, and all subjects were assigned according to criteria (inclusion \& exclusion) and carried out at physiotherapy OPD of CSS Hospital, Meerut. The subjects were equally divided into three groups such as group A (10 subjects, 3 male and 7 female), Group B (10 subjects, 4 male and 6 female) and Group C (10 subjects, 2 male and 8 female). Range of motion of the shoulder joint, ROM and pain was assessed by using the goniometry \& VAS questionnaire respectively. The subjects were reassessed at 3 weeks after completion of intervention. Results: The collected data were of mean and standard deviation of goniometry and SPADI score and has been analyzed using SPSS software version 19.0. The study was done to find out the comparative effect of Mulligan MWM technique versus Kaltenborn mobilization technique on pain and end range of motion of shoulder joint in patients with adhesive capsulitis. Conclusion: This study shows that the effect of Mulligan MWM technique and Kaltensborn mobilization technique was significant in reducing pain and improving end range of motion but on comparison Mulligan 'MWM' was more effective than Kaltenborn mobilization technique.
\end{abstract}

\author{
Sumit Ragav \\ Assistant Professor \\ Subharti College of Physiotherapy \\ Swami Vivekanand Subharti University \\ Delhi-Haridwar Road, Bye Pas Road, NH-58, \\ Meerut-250005 (Uttar Pradesh) India \\ Email: drsumitraghav@gmail.com \\ Anshika Singh \\ Assistant Professor \\ Swami Vivekanand Subharti University \\ Delhi-Haridwar Road, Bye Pas Road, NH-58, \\ Meerut-250005 (Uttar Pradesh) India
}

\begin{abstract}
Key Words: Adhesive Capsulitis, Mulligan 'MWM' technique, Kaltenborn mobilization technique, SPADI and Shoulder joint ROM
\end{abstract}

DOI: $10.18376 / j e s p / 2019 / v 15 / i 1 / 111313$

\section{Introduction}

Adhesive capsulitis is a condition characterized by progressive declination range of motion at the glenohumeral joint due to tightness of capsule. The etiology and pathology of adhesive capsulitis remains unknown (Vermeulen et. al., 2000).But more recent evidence states that adhesive capsulitis is a complex condition caused of inflammation of synoviam and fibrosis in capsule which results in thickening of the inferior capsule lead to contracture. It has been termed "adhesive capsulitis" because of the changes in soft tissues and structure surrounding the joint, such as the posterioinferior joint pouch, the sub-scapularis bursa and the synovial sheath of the long head of biceps 


\section{Journal of Exercise Science \& Physiotherapy Vol. 15 No. 1 (January to June) 2019 ISSN: 0973-2020 (Print) I I OR Impact Factor $=6.502 \quad$ ISSN: 2454-6089 (Online)}

(Robert 1965). The gradual loss of external and internal rotation movement of gleno-humeral joint is the single most important factor in differential diagnosis (Ruiz 2009). Many authorities have been proposed this term including: frozen shoulder, periarthritis, and pericapsulitis. Adhesive capsulitis was firstly described as periarthritis of gleno-humeral joint by Duplay (1872). It described as frozen shoulder by Codman in 1934. But, the term "Adhesive capsulitis" was more precisely given by Neviaser in 1945 that described the pathological changes in the joint capsule and synoviam followed by inflammation and adhesions at open surgery. Patients with Adhesive capsulitis usually present in the sixth decade of life, and its onset is not common before the age of 40yr. Adhesive capsulitis affects about 1 in 50 adults in some stage. The exact incidence and prevalence of it is not known, commonly seen in the age group of 40 to 70 years. The prevalence of adhesive capsulitis in adults of the working age (25-64yr) was found to be $8.25 \%$ in men and $10.15 \%$ in women (Lundberg 1969). Even though adhesive capsulitis is believed to be a self-limiting process; it can be severely disabling for months to years and, as a result, requires appropriate treatment once the diagnosis is made. Initial treatment should include an appropriate physical therapy approach to help regain shoulder joint mobility. For patients in the initial painful or freezing phase, pain relief may be obtained with a course of anti-inflammatory medications, the judicious use of GH joint Corticosteroid injections, or therapeutic modality treatments (Brent and Robert 2011).Various approaches in Physical therapy are available for adhesive capsulitis which helps in maintaining and improving mobility and stability of the shoulder joint (Schenkman and De Cartaya 1987). It includes various grades of mobilizations such as mid-range and end-range mobilizations are suggested by Maitland and Kaltenborn to improve joint mobility and reduce pain (Smith edt al., 2003; Asher 2000; Asher 2000; Vermeulen et al.,2000; Neviaser and Neviaser 1987). To regain the normal extensibility of the shoulder capsule and tight soft tissues, passive stretching of the shoulder capsule and soft tissues by means of mobilization techniques has been recommended, but limited data supporting the use of these techniques are available (Diercks and Stevens.2004; Kaltenborn 2002). One of the joint mobilization technique, Kaltenborn method places special emphasis on translatory linear joint play movements, the convex-concave rule and three-dimensional prepositioning for the joint movement, protecting adjacent joints during procedures, self-treatment and ergonomic principles applied to protect the therapist (Mulligan 2010). Mulligan's MWM is a mobilization technique that used in the management of musculoskeletal disorders. It includes the manual approach form of sustained glide by therapist to joint while concurrent movement of the joint is actively performed by the patient. Mulligan (Susan \& Thomas 2007) originally postulated 'positional fault' to explain the results gained through these techniques. As such, a 'joint tracking mechanism' model is now being used by teachers of the mulligan concept. MWM techniques on the peripheral joints and spine have shown to be effective in reducing pain and increasing range of movement of the joint.

\section{Materials and Method}

The study was a randomized controlled trial with a sample of 30 subjects, 19 were female, 11 were male, and all subjects were assigned according to criteria (inclusion \& exclusion) and carried out at physiotherapy OPD of CSS Hospital, Meerut. The subjects were equally divided into three groups such as group A (10 subjects, 3 male and 7 female), Group B (10 subjects, 4 male and 6 female) and Group C (10 subjects, 2 male and 8 female). Range of motion of the shoulder joint, ROM and pain was assessed by using the goniometry \& VAS questionnaire respectively. The subjects were reassessed at 3 weeks after completion of intervention. After getting their informed consent, the subjects were randomly assigned and allocated in three groups. Subjects for research purpose were selected according to inclusion and exclusion criteria. According to VAS score and goniometry score, the data of pain and range of motion was collected and table of selected variants was prepared and sorting of data was done. The patients in the experimental groups, group A and B 


\section{Journal of Exercise Science \& Physiotherapy Vol. 15 No. 1 (January to June) 2019 ISSN: 0973-2020 (Print) $\quad \mathrm{I}_{2} \mathrm{OR}$ Impact Factor $=6.502 \quad$ ISSN: 2454-6089 (Online)}

subjects followed moist heat pack (MHP), mobilization with movement (MWM) and Kaltenborn mobilization technique respectively. In control Group, group ' $C$ ' subjects advised to perform hot water fomentation and home-based ROM exercises program of the shoulder joint.

In group A, the mobilization with movement (MWM) technique was implemented with three sets of ten repetitions on each treatment occasion for a period of 6 days/ week. In this group, the adjustable couch was used to treat the patients effectively. During MWM, position of the patients was confirmed on the basis of requirement. Techniques of MWM to be performed on the affected shoulder as described by Mulligan.

To improve shoulder abduction ROM: The patient was in relaxed sitting position. A belt was placed around the therapist's hips and the head of the humerus to glide the humeral head appropriately, as the therapist's hand was used over the appropriate aspect of the head of the humerus. A counter pressure was also applied to the scapula with the therapist's other hand. The glide was sustained during slow active shoulder movements to the end of the pain-free range and released after returning to the starting position. Three sets of 10 repetitions will be given followed by 1 minute interval between sets.

To improve shoulder flexion ROM: Subject was in supine lying position. Therapist was standing at the head of the plinth; the therapist grasped the humerus with one hand and the forearm with the other, on the affected side. A downward force was applied along the shaft of the humerus as the patient tried to raise his/her affected arm up to the end of a pain-free range and released after returning to the starting position. Three sets of 10 repetitions will be given followed by 1 minute interval between sets.

To improve shoulder internal rotation ROM: Subject was in standing position with arm in internal rotation and hand far behind his/her back. Therapist was stand on affected side of patient's shoulder. One hand of the therapist was placed on the patient's flexed elbow to give a downward traction force on the humerus while the web space of the other hand was placed in the axilla, obliquely to the stabilized scapula. The patient was asked to internally rotate his/her shoulder with the help of the other hand, while the therapist adducts the patient's upper arm using his/her abdomen. This causes distraction of the humerus laterally. The other hand of the therapist in the axilla acts as a fulcrum. The glides should be painless or slight painful. Three sets of 10 repetitions will be given followed by 1 minute interval between sets.

To improve shoulder external rotation ROM: Subject is in supine position. Therapist was in standing position at the head of the plinth on the affected side. A belt was placed around the bottom of the therapist's foot and just inferior to the head of the humerus to glide the humeral head appropriately, as the therapist's hand is used over the appropriate aspect of the head of the humerus. A downward force was applied along the shaft of the humerus as the patient tries to rotate his/her affected arm up to the end of a pain-free range and released after returning to the starting position. Three sets of 10 repetitions will be given followed by 1 minute interval between sets.

In group B, Kaltenborn mobilization technique was given to the patients. In this group, three sets of ten repetitions on each treatment occasion for a period of 6 days/ week was given for improving range of motion and reducing pain and disability of the shoulder joint. Techniques of joint mobilization to be performed on the affected shoulder as described by Kaltenborn:

Shoulder Traction Technique (for restricted flexion): Subject was in supine lying with affected shoulder was positioned near its available range into flexion with the subject's scapula and thorax stabilized. With a strap placed around the proximal humerus and around the therapist's body, Grade II/III traction movement was performed by leaning backward. Traction was sustained for 15 seconds and repeated 10 times with a gap of 10 seconds.

Shoulder Caudal Glide (for restricted Abduction): Subject was positioned in supine lying; the affected shoulder was positioned near its available range of abduction. Now, by placing one hand 


\section{Journal of Exercise Science \& Physiotherapy Vol. 15 No. 1 (January to June) 2019 ISSN: 0973-2020 (Print) I I OR Impact Factor $=6.502 \quad$ ISSN: 2454-6089 (Online)}

over the humerus just distal to the joint space, Grade II/III caudal glide was to be given by shifting the body forward. Glide was sustained for 15 seconds; this was repeated 10 times with a gap of 10 seconds.

Shoulder Ventral Glide (for External rotation): Subject was in prone lying position with a towel roll under the coracoid process and the arm beyond the edge of the couch. Now, by grasping the humeral head with hypothenar eminence of one hand and supporting the arm near the elbow with the other, Grade II/III ventral glide was performed with the shoulder positioned near its available ROM in external rotation. Glide was sustained for 15 seconds; this was repeated 10 times with a gap of 10 seconds.

Shoulder Dorsal Glide (for internal rotation): Subject to be positioned in supine with the scapula stabilized with a towel roll. Now, by holding the humerus against the body with both hands and gripping the humeral head just distal to the joint space with the hypothenar eminence with the hand closer to the joint, Grade II/III dorsal gliding movement to the joint is to be given. Glide is sustained for 15 seconds; this was repeated 10 times with a gap of 10 seconds.

Depending upon the pattern of range of motion restriction, suitable techniques are to be used.

In both experimental groups, the protocol was used to accommodate the tolerance level of patients with various degrees of motion. Patients were also instructed to perform home exercises program in three sets of 12 repetitions, twice daily. In control group, the home exercise program was advised to those patients who did not come to the OPD on regular basis. The exercise program consisting of range of motion exercises and stretching exercises was taught to the patients. The range of motion exercises consisted of wall ladder exercises and pendulum exercises. The participants were instructed to perform a total of 10-12 repetitions of each movement. Each repetition lasted 6 seconds with an interval of approximately 3 seconds. All data were seen in two consecutive visits; initial evaluation on $1^{\text {st }}$ day and last on $21^{\text {th }}$ day respectively but were instructed to follow the home exercise program for a total of 3 weeks. The home exercise program consisted of hot fomentation along with range of motion exercises of shoulder joint. All statistical analysis was obtained using SPSS version 20.0. Demo graphic data of the patients including range of motion and pain were summarized. The dependent variables for the statistical analysis were SPADI and Goniometry score. A base line data was taken and analyze. ANOVA (F-test) was used in this study. A level of significance $5 \%$ was used to determine the statistical significance.

\section{Results}

This study provides data for pain and range of motion of persons who had post traumatic shoulder joint stiffness. The data is sparse in between 40-60 age groups since it was convenient to find people in this age group who could fit the inclusion criteria in this study. In this study, data shows there is decreasing in VAS score and increasing range of motion (ROM) score in patients with Adhesive capsulitis. Both treatment Mulligan 'MWM' and Kaltenborn Mobilization technique are effective in reducing pain and improving range of motion of the shoulder joint. But MWM is more effective than Kaltenborn and conventional exercises program in reducing pain and joint stiffness. Data of ROM and VAS of three Groups i.e. Mulligan 'MWM', Kaltenborn Mobilization technique and conventional exercises program for pre and post interventional study are expressed in terms of mean, S.D and S.E.M is shown in table-1, 2,3,4,5 and 6 respectively. Further application of ANOVA (F-test) to find the significant difference between pre and post intervention study in Mulligan 'MWM', Kaltenborn Mobilization technique and conventional exercises program, which revealed significance difference for the 10 patients each group individual at $5 \%$ level of significance.In both groups, $\mathrm{p}$-value was significant i.e., $\mathrm{p}<0.05$ with ROM and VAS score $(0.0000)$ and (0.0000) respectively. The 3-weeks protocol of Mulligan 'MWM', Kaltenborn Mobilization technique and conventional exercises program showed difference in three groups individually in order to improving range of motion and also decreasing the pain but both experimental groups A 
and B, 'MWM' and Kaltenborn mobilization technique showed statistically more significant difference in decreasing pain, and improving range of motion. But on comparison between experimental groups, the effect of Mulligan's technique was more statistically significant in reducing pain and improving $\mathrm{ROM}$.

Table 1. Pre and Post Score of Flexion Range

\begin{tabular}{|c|c|c|c|c|c|c|c|c|}
\hline Groups & \multicolumn{2}{|c|}{ Mean } & \multicolumn{2}{c|}{ S.D } & \multicolumn{2}{c|}{ S.E.M } & F-Test & P-value \\
\hline & pre & post & pre & post & pre & post & & \\
\hline Group A & 143.50 & 156 & 14.347 & 34.944 & 4.537 & 11.050 & 1.095 & .309 \\
\hline Group B & 130 & 143 & 7.817 & 9.487 & 3.000 & 2.472 & 11.184 & .004 \\
\hline Group C & 141 & 144.50 & 11.255 & 12.122 & 3.559 & 3.833 & .448 & .512 \\
\hline
\end{tabular}

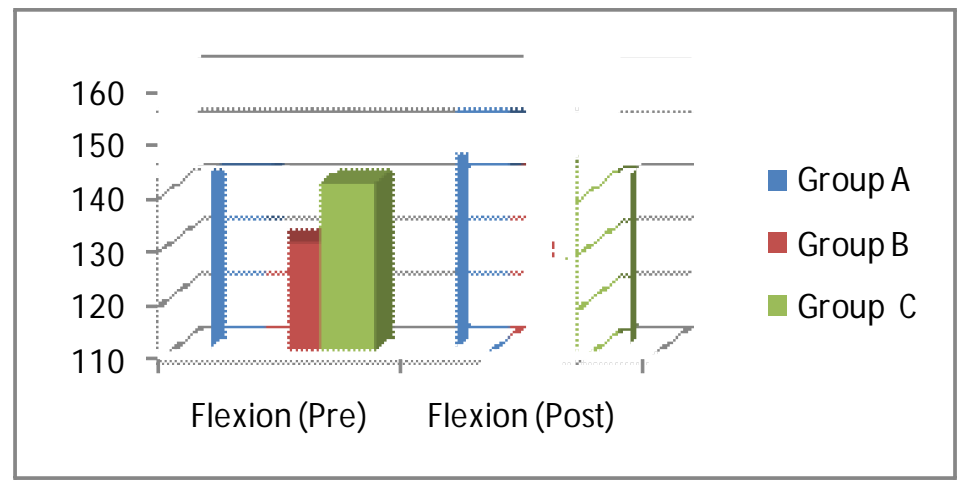

Figure 1. Pre and post score of Flexion Range

Table 2. Pre and Post Score of Extension Range

\begin{tabular}{|l|c|c|c|c|c|c|c|c|}
\hline Groups & \multicolumn{2}{|c|}{ Mean } & \multicolumn{2}{c|}{ S.D } & \multicolumn{2}{c|}{ S.E.M } & F-Test & P-value \\
\hline & pre & post & pre & post & pre & post & & \\
\hline Group A & 41.50 & 54.50 & 7.091 & 5.986 & 2.242 & 1.893 & 19.626 & .000 \\
\hline Group B & 42.50 & 49.50 & 5.401 & 6.433 & 1.708 & 2.034 & 6.945 & .017 \\
\hline Group C & 48.50 & 51.50 & 7.835 & 7.091 & 2.478 & 2.242 & .806 & .381 \\
\hline
\end{tabular}


Journal of Exercise Science \& Physiotherapy Vol. 15 No. 1 (January to June) 2019

ISSN: 0973-2020 (Print) $\quad \mathrm{I}_{2} \mathrm{OR}$ Impact Factor $=6.502 \quad$ ISSN: 2454-6089 (Online)

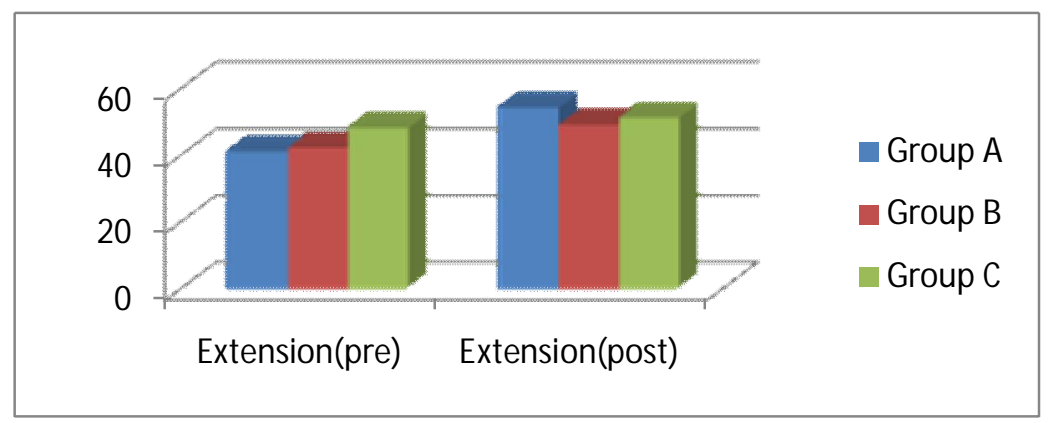

Figure 2. Pre and post score of Extension Range

Table 3. Pre and Post Score of Abduction Range

\begin{tabular}{|c|c|c|c|c|c|c|c|c|}
\hline Groups & \multicolumn{2}{|c|}{ Mean } & \multicolumn{2}{c|}{ S.D } & \multicolumn{2}{c|}{ S.E.M } & F-Test & P-value \\
\hline & pre & post & pre & post & pre & post & & \\
\hline Group A & 110.50 & 157.00 & 12.349 & 19.607 & 3.905 & 6.200 & 40.270 & .000 \\
\hline Group B & 112.00 & 141.50 & 9.189 & 11.068 & 2.906 & 3.500 & 42.052 & .000 \\
\hline Group C & 116.50 & 127.50 & 9.733 & 8.580 & 3.078 & 2.713 & 7.188 & .015 \\
\hline
\end{tabular}

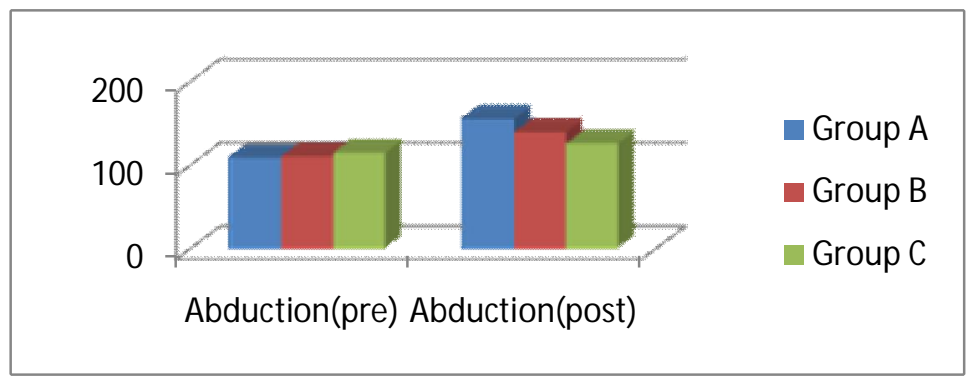

Figure 3. Pre and post score of Abduction Range

Table 4. Pre and Post Score of Medial Rotation Range

\begin{tabular}{|c|c|c|c|c|c|c|c|c|}
\hline Groups & \multicolumn{2}{|c|}{ Mean } & \multicolumn{2}{c|}{ S.D } & \multicolumn{2}{c|}{ S.E.M } & F-Test & P-value \\
\hline & pre & post & pre & post & pre & post & & \\
\hline Group A & 38 & 59 & 8.233 & 8.097 & 2.603 & 2.560 & 33.075 & .000 \\
\hline Group B & 38.50 & 50.50 & 5.798 & 7.619 & 1.833 & 2.409 & 15.709 & .001 \\
\hline Group C & 45.50 & 47.00 & 5.986 & 4.216 & 1.893 & 1.333 & .420 & .525 \\
\hline
\end{tabular}


Journal of Exercise Science \& Physiotherapy Vol. 15 No. 1 (January to June) 2019

ISSN: 0973-2020 (Print) $\quad \mathrm{I}_{2} \mathrm{OR}$ Impact Factor $=6.502 \quad$ ISSN: 2454-6089 (Online)

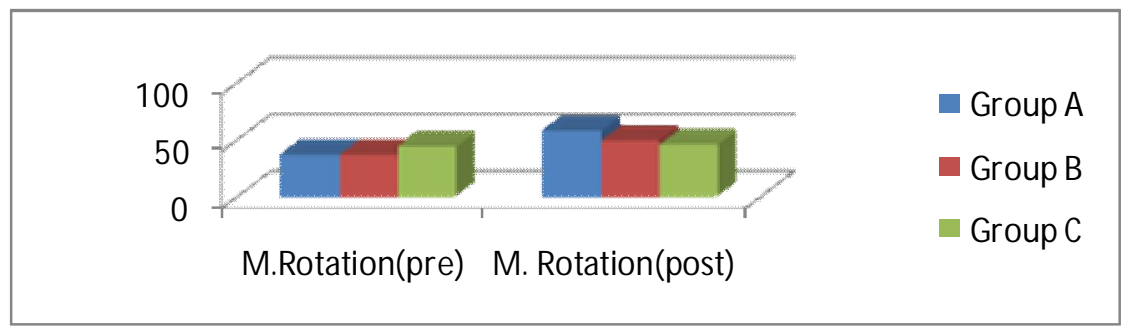

Figure 4. Pre and post score of Medial Rotation Range

Table 5. Pre and Post Score of Lateral Rotation Range

\begin{tabular}{|c|c|c|c|c|c|c|c|c|}
\hline Groups & \multicolumn{2}{|c|}{ Mean } & \multicolumn{2}{c|}{ S.D } & \multicolumn{2}{c|}{ S.E.M } & F-Test & P-value \\
\hline & pre & post & pre & post & pre & post & & \\
\hline Group A & 45 & 71 & 9.428 & 8.097 & 2.981 & 2.560 & 43.770 & .000 \\
\hline Group B & 43.50 & 59.60 & 4.126 & 8.317 & 1.302 & 2.630 & 29.729 & .000 \\
\hline Group C & 48 & 51 & 7.888 & 8.756 & 2.494 & 2.769 & .648 & .431 \\
\hline
\end{tabular}

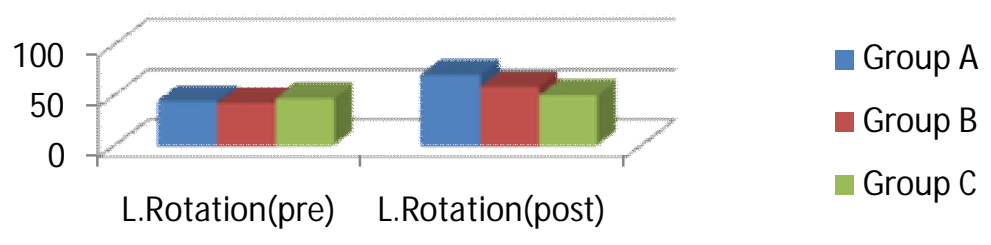

Figure 5. Pre and post score of Lateral Rotation Range

Table 6. Pre and Post score of VAS

\begin{tabular}{|l|c|c|c|c|c|c|c|c|}
\hline Groups & \multicolumn{2}{|c|}{ Mean } & \multicolumn{2}{c|}{ S.D } & \multicolumn{2}{c|}{ S.E.M } & F-Test & P-value \\
\hline & pre & post & pre & post & pre & post & & \\
\hline Group A & 4.70 & 1.00 & .675 & .667 & .213 & .211 & 152.111 & .000 \\
\hline Group B & 4.80 & 1.80 & .327 & .200 & .327 & .200 & 61.364 & .000 \\
\hline Group C & 4.20 & 2.30 & .789 & .675 & .249 & .213 & 33.495 & .000 \\
\hline
\end{tabular}

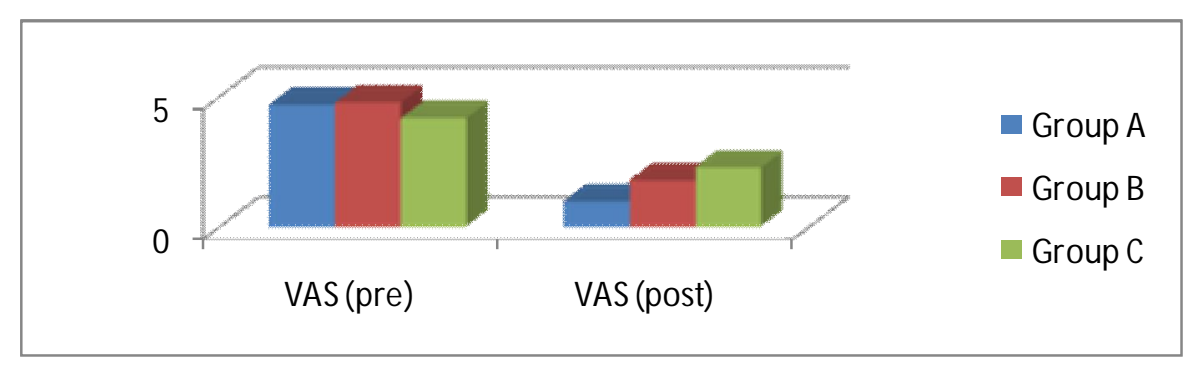

Figure 6. Pre and post score of VAS 


\section{Journal of Exercise Science \& Physiotherapy Vol. 15 No. 1 (January to June) 2019 \\ ISSN: 0973-2020 (Print) I I OR Impact Factor $=6.502 \quad$ ISSN: 2454-6089 (Online)}

\section{Discussion}

In this study, data shows there is decreasing in VAS score and increasing range of motion (ROM) score in patients with Adhesive capsulitis. Both treatment Mulligan 'MWM' and Kaltenborn Mobilization technique are effective in reducing pain and improving range of motion of the shoulder joint. But MWM is more effective than Kaltenborn and conventional exercises program in reducing pain and joint stiffness. The 3-weeks protocol of Mulligan 'MWM', Kaltenborn Mobilization technique and conventional exercises program showed difference in three groups individually in order to improving range of motion and also decreasing the pain but both experimental groups A and B, 'MWM' and Kaltenborn mobilization technique showed statistically more significant difference in decreasing pain, and improving range of motion. But on comparison between experimental groups, the effect of Mulligan's technique was more statistically significant in reducing pain and improving ROM. Ujwal et. al., (2017) did a study on the effectiveness of MWM in adhesive capsulitis of shoulder joint. The purpose of their study, the ability to perform activities of daily living is important in maintaining independence for the older adult. With aging, the loss of physiological reserve increases the risk of secondary disorders alike adhesive capsulitis of shoulder. In their study the patients diagnosed with adhesive capsulitis and referred to physiotherapy were recruited $(n=30)$. These patients were divided randomly using simple random sampling into Group A (15) undergoing MWM intervention and Group B (15) undergoing supervised exercises only. All the patients underwent intervention for 1 week and were assessed pre-post intervention for range of motion, pain using Numerical Pain Rating Scale (NPRS); and Shoulder Pain and Disability Index (SPADI). The data collected was analyzed using unpaired t-test. Total 30 patients (16 males and 14 females) with a mean age of 56.3 7.92 years underwent intervention. Pain score on NPRS improved from $7.93 \pm 0.88$ to3.4 \pm 1.24 in Group A ( $<<0.005$ ) as compared to $8.06 \pm 1.09$ to $6.4 \pm 1.2$ in Group B $(\mathrm{p}<0.005)$. The SPADI score improved from $91.7 \pm 6.90$ to $35.26 \pm 3.45$ in Group A $(\mathrm{p}<0.005)$ as compared to92.4 \pm 4.15 to $69.53 \pm 6.7$ in Group B $(\mathrm{p}<0.005)$. Their study concluded that Movement with mobilization proved to be a better technique for improving range of motion and pain in adhesive capsulitis of shoulder. Another study which was conducted by Henricus e.t al., (2000) on End-range mobilization techniques in Adhesive capsulitis of the shoulder joint: A multiple- subject case report. The purpose of this case report is to describe the use of end-range mobilization techniques in the management of patients with adhesive capsulitis. In their case report 4 male and 3 female with Adhesive capsulitis of the glenohumeral joint were treated with end-range mobilization techniques, twice a week for 3 months. In their study, there was significant difference in order to decrease pain and improve mobility at glenohumeral joint. There seemed to be a role for intensive mobilization techniques in the treatment of adhesive capsulitis. Controlled studies regarding the effectiveness of end range mobilization techniques in the treatment of adhesive capsulitis are warranted. Gui et. al., (2015) did a study on comparison of Maitland and Kaltenborn mobilization techniques for improving shoulder pain and range of motion in frozen shoulder. Total no 20 subjects were participated in their study. The subjects were equally divided into two groups. One group received Maitland mobilization technique and other group received Kaltenborn mobilization technique. Both groups exhibited significant decreased in pain post- intervention. Moreover, the range of motion of internal and external rotation increased significantly post-intervention in both groups. However, there was no significant difference between groups with respect to decrease pain and improve range of motion.Both techniques, Maitland and Kaltenborn mobilization technique were effective for improving range of motion and decreasing pain in patients with frozen shoulder. There is less empirical data available related to this study which shows a significant effect of Kaltenborn mobilization technique in reducing pain and improving ROM in patients Adhesive Capsulitis of shoulder joint. But in this study the significant difference showed in pre to post ROM and VAS score in three groups. Finally, 


\section{Journal of Exercise Science \& Physiotherapy Vol. 15 No. 1 (January to June) 2019 ISSN: 0973-2020 (Print) I I OR Impact Factor $=6.502 \quad$ ISSN: 2454-6089 (Online)}

Group A received Mulligan's technique (MWM) showed statistically significant difference in order to decrease pain and in improving ROM.

\section{Conclusion}

Study conclude that the difference from 1 st to $21^{\text {th }}$ day in ROM and VAS score in three groups/therapies which shows that three groups A,B and C i.e. Mulligan's technique (MWM), Kaltenborn mobilization technique and conventional exercises reduced pain and improved range of motion. But Mulligan's technique (MWM) showed statistically more significant difference in ROM and VAS score in order to decrease pain and in improving ROM.

\section{References}

Asher S.N. 2000.Frozen Shoulder Syndrome. Osteopath Dec 2000.

Brent S. Brotzman and Robert C. Manske.2011. In: Clinical Orthopedic Rehabilitation: An Evidence- Based Approach $3^{\text {rd }}$ edition;608.

Codman E.A. 1934. Tendinitis of the Short Rotators. In: The Shoulder: Rupture of the Supraspinatus Tendon and Other Lesions in or about the Subacromial Bursa. Boston MA: Thomas Todd.

Diercks R.L.and Stevens M.2004. Gentle Thawing of the Frozen Shoulder: A Prospective Study of Supervised Neglect versus Intensive Physical Therapy in Seventy-Seven Patients with Frozen Shoulder Syndrome followed up for two years. J Shoulder Elbow Surg.;13:499-502.

Duplay E.S. De la.1872. Periarthritescapulo-humeraleet des raideurs de I eupaule qui ensont la consequence. Arch Gen Med ;20:513-542.

Gui DO MOON et. al.,2015. Comparison of Maitland and Kaltenborn Mobilisation Techniques for Improving Shoulder Pain and Range of Motion in Frozen Shoulder. J. Pys.Ther.Sci.27: 1391-1395.

Henricus M. Vermeulen et. al.,2000. End-Range Mobilization Techniques in Adhesive Capsulitis of the Shoulder Joint: A Case Report. Physical Therapy December;80(12); 1204-1213.

Kaltenborn F.M.2002. Manual Mobilization of Joints: Joint Examination and Basic Treatment, 6th Edition, Norli Oslo Norway.

Lundberg B.J.1969. The Frozen Shoulder. Acta Orthop Scand; 119, 1-59.

Mulligan B.R. 2010.Manual Therapy: NAGS ,SNAGS, MWM's etc. 6th ed. Wellington, New Zealand. Jack Miller:Plane View Services Ltd.

Neviaser J.S.1945. Adhesive capsulitis of the shoulder. J Bone Joint Surg ;27:211-22.

Neviaser R.j. and Neviaser T.I.1987.The Frozen Shoulder: Diagnosis and Management. Clin Orthop 223:5964.

Robert L. Samilson.1965. Shoulder Pain: Diagnosis and treatment of injuries to soft tissues. California Medical Association. Jan;102, 1:23-25.

Ruiz J.2009. Positional Stretching of the Coracohumeral Ligament on a Patient with Adhesive Capsulitis: A Case Report. The Journal of Manual \& Manipulative Therapy;17 (1): 58-63.

Schenkman M., and De Cartaya V.R.1987. Kinesiology of the Shoulder Complex. J Orthop Sports Phys Ther ;8:438-450.

Smith L.L., Burnet S.P., McNeil J.D. 2003.Musculoskeletal Manifestations of Diabetes mellitus. Br J Sport Med ;37:30-35.

Susan B. O'Sullivan \& Thomas J. Schmitz.2007. In: Physical Rrehabilitation, chapter 11, 5th edition, Published by Jaypee Brothers' Medical Publishers' Ltd., page no. 381.

Ujwal L. Yeole, Pratiksha D. Dighe et. al.2017. Effectiveness of Movement with Mobilization in Adhesive Capsulitis of Shoulder: Randomized Controlled Trial. Indian Journal of Medical Research and Pharmaceutical Sciences; February;4(2); 1-8.

Vermeulen H. M., Obermann W. R., Burger B. J., Kok G. J., Rozing P. M. and Van den Ende C.2000. EndRange Mobilization Techniques in Adhesive Capsulitis of the Shoulder Joint: A Multiple-Subject Case Report. Phys ther. 80:1204-1213.

Vermeulen H.M., Obermann W.R., Burger B.J.,et al.2000. End-Range Mobilization Techniques in Adhesive Capsulitis of the Shoulder Joint: a Multiple-Subject Case Report. Phys Ther ;80:1204-1213.

\section{Conflict of Interest: None declared}

